\title{
Experimentelle und semi-empirische Protonen-Hyperfein- kopplungen von 1.3-Benzodioxol-Radikal-Kationen und ihre Korrelation mit Reaktivitäten in elektrophilen Substitutionen *
}

Jörg Fleischhauer, Sun Ma und Wolfgang Schleker

Lehr- und Forschungsgebiet Theoretische Chemie der RWTH Aachen

Klaus Gersonde und Hans Twilfer

Lehr- und Forschungsgebiet Physikalische Chemie der Proteine der RWTH Aachen

Franz Dallacker

Lehr- und Forschungsgebiet Organische Chemie und Abteilung Chemie für Mediziner der RWTH Aachen

Z. Naturforsch. 37a, 680-687 (1982); eingegangen am 4. Mai 1982

Herrn Professor Dr. Hermann Stetter zu seinem 65. Geburtstag gewidmet

Experimental and Semi-Empirical Proton Hyperfine Splitting Constants of 1.3-Benzodioxole Radical Cations and their Correlation with Reactivities in Electrophilic Substitutions

The solution ESR X-band spectra of 1.3-benzodioxole (methylenedioxybenzene) (1), benzo[1.2-d:4.5-d'] bis[1.3]dioxole (2), benzo[1.2-d:3.4- $\left.\mathrm{d}^{\prime}\right]$ bis[1.3]dioxole (3) and benzotris[1.3]dioxole (4) have been measured at room temperature. Hyperfine splitting constants of the aromatic ring protons of the compounds 1-3 have been determined. They have been correlated with the HOMO orbital densities of the adjacent carbon atoms and exhibit sufficient correspondence with the reactivities of these compounds in electrophilic substitution reactions. The splitting constants of all protons of the compounds 1-4 have been calculated by the INDO and HMO methods and have been compared with the experimental hyperfine values. By perturbation theory applied to the interactions between the $\pi$-MO's of benzene and the dioxole fragment one can explain and understand the magnitudes of the experimental hyperfine splitting constants of the hydrogen atoms of these compounds. In particular, the hyperfine values of the methylene protons calculated by the HMO method are in good agreement with the experimental data.

\section{Einleitung}

Abkömmlinge des 1.3-Benzodioxols (Methylendioxybenzols) (1) sind als sekundäre Metaboliten in der Pflanzenwelt weit verbreitet. Dagegen scheinen das Benzo[1.2-d:4.5-d']bis[1.3] dioxol (2) [1], das Benzo[1.2-d:3.4-d'] bis[1.3]dioxol (3) [2] und das Benzotris [1.3] dioxol (4) [3] nur theoretisches Interesse zu beanspruchen. Wenn sie auch bisher noch nicht in natürlichen Materialien aufgefunden wurden, so besitzen doch zumindest $\mathbf{2}$ und $\mathbf{3}$ zur Darstellung chinoider Systeme eine praktische Bedeutung.

Von den Verbindungen 1 bis 4 tragen nur 1, 2 und $\mathbf{3}$ aromatische Wasserstoff-Atome. Die Verbindung 1 zeigt eine hohe $S_{\mathrm{E}}$-Reaktivität in $\operatorname{der} \beta$-Posi-

\footnotetext{
* Auszugsweise vorgetragen auf der Chemiedozententagung in Kaiserslautern am 25. 3. 1982.

Sonderdruckanforderungen an Prof. Dr. J. Fleischhauer, Lehr- und Forschungsgebiet Theoretische Chemie der RWTH Aachen, Prof.-Pirlet-Straße 1, D-5100 Aachen.
}

tion. In hohen Ausbeuten lassen sich z. B. die Monound Dibrom- oder die Mono- und Dinitroverbindungen darstellen.<smiles>Oc1c(O)c2c(c(P)c1P)OCO2</smiles><smiles>[CH]1Oc2cc3c(cc2O1)O[CH]O3</smiles>

3

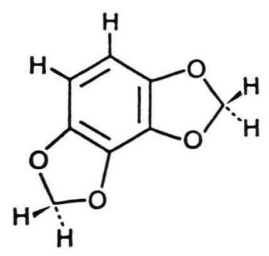

4

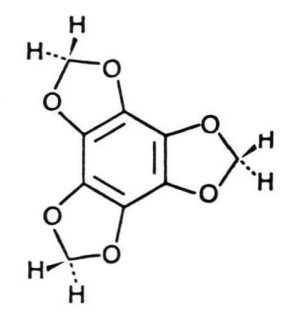

0340-4811 / 82 / 0700-0680 $\$ 01.30 / 0$. - Please order a reprint rather than making your own copy. 
Ein Vergleich von 1 und $\mathbf{3}$ ergibt, daß 3 eine höhere $S_{\mathrm{E}}$-Reaktivität besitzt. So läßt sich z. B. nur 3 einer direkten Formylierung nach Vilsmeier unterziehen. Der Aldehyd entsteht dabei in guter Ausbeute [3].

Dagegen ist die $S_{\mathrm{E}}$-Reaktivität von 2 extrem gering. Hier sind nur direkte Metallierungen z. B. durch Verwendung von Butyllithium möglich. Die Einwirkung von Brom oder Salpetersäure führt unter drastischen Bedingungen zu Molekülveränderungen. Dabei treten in der Regel Ätherspaltungen ein.

Um das unterschiedliche Reaktionsverhalten der Verbindungen $\mathbf{1}-\mathbf{3}$ zu verstehen, wurden die ESRSpektren ihrer Radikal-Kationen aufgenommen, um aus den Aufspaltungskonstanten der aromatischen Ring-Protonen die $\pi$-Homodichten an den betreffenden Zentren zu ermitteln. Diese sind im Rahmen des Grenzorbitalmodells [4,5] mitbestimmend für die unterschiedliche Reaktivität in einer elektrophilen Substitutionsreaktion.

Wie bereits Dixon und Murphy [6] gefunden haben, sind die Hyperfeinaufspaltungskonstanten der Methylenprotonen bei $\mathbf{1}$ außergewöhnlich groß. Sie erklären diesen Befund durch einen Hyperkonjugationseffekt. Es interessierte daher, neben 1-3 auch noch die Verbindung 4 zu betrachten, um zu untersuchen, ob man mit diesem Modell auch den beobachteten Gang der Aufspaltungskonstanten der Methylenprotonen von 1-4 verstehen kann.

Weiterhin sollte untersucht werden, inwieweit die Übereinstimmung zwischen experimentellen und semi-empirisch berechneten Aufspaltungskonstanten (INDO, HMO) bei diesen Verbindungen durch die Wahl verschiedener Parametersätze, Auswertungsverfahren (z.B. Annihilierung des Quartettanteils der UHF-Wellenfunktion), sowie Verwendung verschiedener Geometrien optimiert werden kann.

\section{ESR-Spektren der Radikal-Kationen der Verbindungen 1-4}

\subsection{Methylendioxybenzol (1)}

Die gefundenen Aufspaltungskonstanten sind etwas kleiner als in [6] beschrieben (s. Abb. 1 und Tabelle 6). Die kleinste Aufspaltung der a-Protonen $(0,04 \mathrm{mT}[6])$ konnte wegen der zu hohen Modulationsamplitude nicht aufgelöst werden. Die relativ große Aufspaltungskonstante $a_{\mathrm{H}_{\beta}}$ für das $\beta$ Proton $(0,481 \mathrm{mT} ; 0,49 \mathrm{mT}$ [6]) macht die hohe

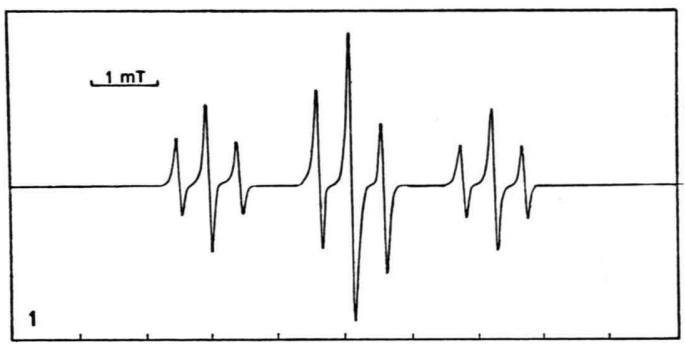

Abb. 1. ESR-Spektrum des Radikal-Kations des Methylendioxybenzols (1) (Exp. Bedingungen s. Tab. 4 und 5).

$S_{\mathrm{E}}$-Reaktivität von 1 verständlich, da wegen der McConnell-Beziehung die $\pi$-HOMO-Dichte am $\mathrm{C}_{\beta}$ dem Wert von $a_{\mathrm{H}_{\beta}}$ proportional ist. Auffallend groß ist, wie bereits von Dixon und Murphy gefunden, die Aufspaltungskonstante der Methylenprotonen $(2,142 \mathrm{mT} ; 2,19 \mathrm{mT}[6])$.

\subsection{Benzo[1.2-d:4.5-d']bis[1.3] dioxol (2)}

Aus der Strukturformel muß man zwei Sätze äquivalenter Protonen erwarten, nämlich die vier Protonen der beiden Dioxol-Gruppen und die zwei identischen Ring-Protonen. Somit sollte das Spektrum fünf Tripletts mit den relativen Intensitäten von $1: 4: 6: 4: 1$ zeigen, was auch gefunden wird (s. Abb. 2 und Tabelle 6). Die extrem geringe $S_{\mathrm{E}}$-Reaktivität korrespondiert mit einem relativ kleinen Wert für die Aufspaltungskonstante der aromatischen Ring-Protonen $(0,102 \mathrm{mT})$. Auffallend ist weiterhin, daß die Aufspaltungskonstante der Methylenprotonen $(1,157 \mathrm{mT})$ im Vergleich zu 1 fast halbiert ist.

\subsection{Benzo[1.2-d:3.4- $\left.d^{\prime}\right]$ bis [1.3]dioxol (3)}

Dieses Derivat ist das Isomere der zuvor beschriebenen Verbindung und sollte daher ein ähnliches

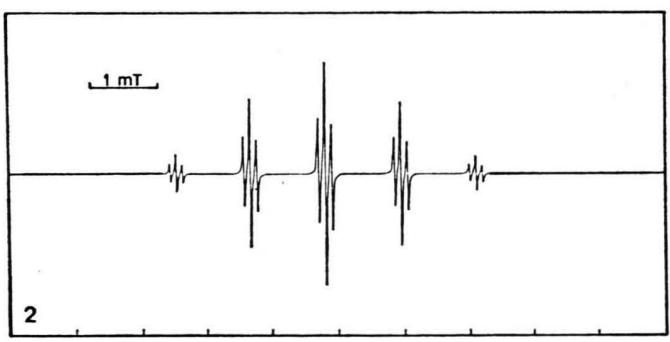

Abb. 2. ESR-Spektrum des Radikal-Kations des Benzo[1.2d:4.5-d']bis-[1.3]dioxols (2) (Exp. Bedingungen s. Tab. 4 und 5). 


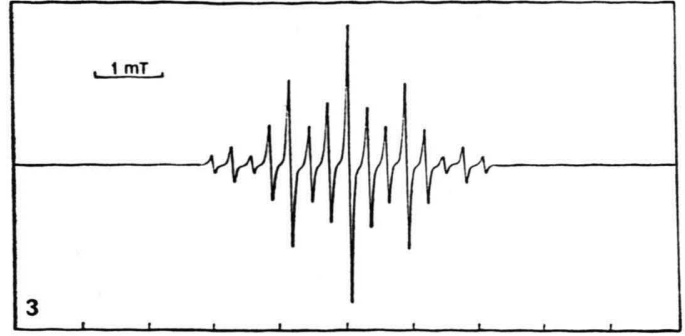

Abb. 3. ESR-Spektrum des Radikal-Kations des Benzo[1.2-d :3.4-d']bis-[1.3]dioxols (3) (Exp. Bedingungen s. Tab.4 und 5).

ESR-Spektrum zeigen. Wie Abb. 3 zeigt, werden ebenfalls fünf Tripletts gefunden, die jedoch wegen der kleineren Aufspaltung der Methylenprotonen $(0,891 \mathrm{mT})$ und der größeren Aufspaltung der Ring-Protonen $(0,298 \mathrm{mT})$ nicht so deutlich separiert sind. Die beobachtete größere $S_{\mathrm{E}}$-Reaktivität von $3 \mathrm{im}$ Vergleich zu 1 kann hier nicht allein durch die Größe der Aufspaltungskonstanten der RingProtonen verstanden werden, da diese bei 3 kleiner $(0,298 \mathrm{mT})$ als bei $1(0,481 \mathrm{mT})$ ist. Eine mögliche Erklärung ist jedoch, daß die Energie des $\pi$ HOMO's von 3 positiver $(\alpha+0,594 \beta)$ als die von $1(\alpha+0,688 \beta)$ ist und damit näher bei der des LUMO's des jeweils angreifenden Elektrophils liegt. Interessant ist weiterhin, daß die Aufspaltungskonstante der Methylenprotonen im Vergleich zu der von 2 noch etwas kleiner ist (s. Tabelle 6).

\subsection{Benzotris [1.3] dioxol (4)}

In Abb. 4 ist das ESR-Spektrum des Benzotris[1.3] dioxols dargestellt. Wegen der sechs identischen Methylenprotonen sind sieben Resonanzlinien zu erwarten. Die Aufspaltungskonstante $a_{\mathrm{CH} 2}$ beträgt nur noch $0,544 \mathrm{mT}$.

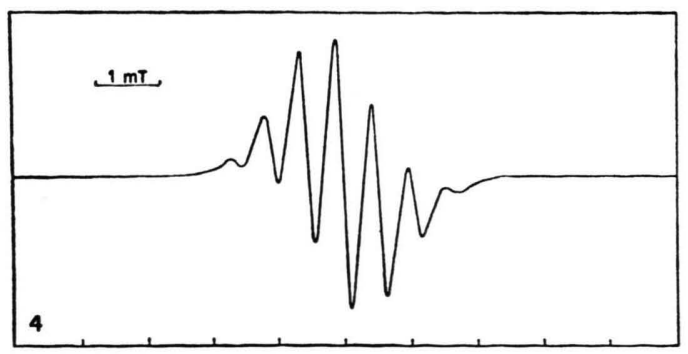

Abb. 4. ESR-Spektrum des Radikal-Kations des Benzotris[1.3]dioxols (4) (Exp. Bedingungen s. Tab. 4 und 5).

\section{Berechnung der Aufspaltungskonstanten der Verbindungen 1-4 mit semi-empirischen Methoden}

\subsection{INDO-Methode}

Die Geometrie des Methylendioxybenzols 1 wurde aus Standardwerten mit teilweiser Optimierung des Dioxol-Rings erzeugt [7] und dann für die Moleküle 2-4 entsprechend weiterverwendet.

Für die Rechnungen wurden sowohl die SlaterCondon-Parameter von Pople [8] als auch die von Kaufman [9] benutzt. Die Aufspaltungskonstanten wurden aus den Spin-Dichten an den H-Atomen mit den in der Arbeit von Tino und Klimo [10] angegebenen Proportionalitätskonstanten $K$ für die verschiedenen Parametersätze ermittelt.

Wie man Tab. 1 entnimmt, liegt der mit diesen UHF-Wellenfunktionen errechnete Erwartungswert von $\hat{S}^{2}$ deutlich oberhalb von 0,75 . Der Quartettanteil wurde daher nach Amos und Snyder [11] herausprojiziert. In Tab. 1 sind die resultierenden Aufspaltungskonstanten vor und nach der QuartettAnnihilierung angegeben.

Tab. 1. Theoretische Hyperfeinaufspaltungen in $\mathrm{mT}$, berechnet mit Hilfe der INDO-Methode, und Erwartungswerte von $\widehat{S^{2}}$.

\begin{tabular}{|c|c|c|c|c|c|}
\hline $\begin{array}{l}\text { Me- } \\
\text { thode }\end{array}$ & 1 & 2 & 3 & 4 & exp. \\
\hline \multicolumn{6}{|c|}{ Verbindung 1} \\
\hline$\left\langle\widehat{S^{2}}\right\rangle$ & 0,7790 & 0,7504 & 0,7827 & 0,7506 & \\
\hline$a_{\mathrm{CH}_{2}}$ & 2,1560 & 2,1122 & 2,0256 & 2,2761 & 2,142 \\
\hline$a_{\mathrm{H}_{\alpha}}$ & 0,0618 & 0,0290 & 0,1165 & 0,0613 & 0,0 \\
\hline$a_{\mathrm{H}_{\beta}}$ & $-0,2358$ & $-0,1016$ & $-0,3545$ & $-0,1763$ & (-) 0,481 \\
\hline
\end{tabular}

Verbindung 2

\begin{tabular}{|c|c|c|c|c|c|}
\hline$\left\langle\widehat{S^{2}}\right\rangle$ & 0,7928 & 0,7510 & 0,8003 & 0,7514 & \\
\hline$a_{\mathrm{CH}_{2}}$ & 0,5109 & 0,5662 & 0,4963 & 0,6208 & 1,157 \\
\hline$a_{\mathrm{H}}$ & 0,2021 & 0,0871 & 0,3545 & 0,1763 & 0,102 \\
\hline \multicolumn{6}{|c|}{ Verbindung 3} \\
\hline & 0,7847 & 0,7501 & 0,7884 & 0,7502 & \\
\hline & 0,5109 & 0,5589 & 0,4861 & 0,6131 & 0,891 \\
\hline & $-0,1460$ & $-0,0653$ & $-0,2026$ & $-0,0996$ & 0,298 \\
\hline
\end{tabular}

Verbindung 4

\begin{tabular}{lrrrrr}
$\left\langle\widehat{S^{2}}\right\rangle$ & 0,7870 & 0,7503 & 0,7920 & 0,7505 & \\
$a_{\mathrm{CH}_{2}}$ & 0,2564 & 0,3097 & 0,2414 & 0,3398 & 0,544 \\
$K / \mathrm{mT}$ & 56,147 & 72,585 & 50,639 & 76,638 & \\
\hline
\end{tabular}

Methoden: 1: nach Pople, ohne Annihilierung; 2: nach Pople, mit Annihilierung; 3: nach Kaufman, ohne Annihilierung; 4: nach Kaufman, mit Annihilierung. 
Die quantitative Übereinstimmung der berechneten und experimentellen Aufspaltungskonstanten ist recht unbefriedigend. Die Annihilierung bringt keine Verbesserung [12]. Der beobachtete Gang der experimentell ermittelten Aufspaltungskonstanten der Methylenprotonen wird von den Methoden 2-4 richtig wiedergegeben, aber die quantitative Übereinstimmung ist nur im Fall von Verbindung 1 befriedigend. Der experimentelle Gang bezüglich der Hyperfeinaufspaltung für die aromatischen Protonen wird nicht durch die Theorie bestätigt. Nur im Fall von Verbindung 1 wird deutlich, daß der Betrag von $a_{\mathrm{H}_{\alpha}}$ kleiner als der von $a_{\mathrm{H}_{\beta}}$ ist.

\subsection{Abhängigkeit der berechneten Aufspaltungs- konstanten von der Geometrie}

Am Beispiel des Methylendioxybenzols 1 wurde der Einfluß der benutzten Geometrie auf die berechneten Spin-Dichten untersucht. Das neutrale Molekül (1a) und das Radikal-Kation (1b) wurden mit der MINDO/3-Methode [13] optimiert, wobei alle Bindungsabstände, -winkel und Diederwinkel frei variabel waren. Nur der Benzolring wurde als planar festgesetzt. Die so ermittelten Konformationen waren die Grundlage erneuter INDO-Berechnungen, deren Ergebnisse in Tab. 2 aufgelistet sind.

Wie die Ergebnisse zeigen, erhält man eine etwas bessere Übereinstimmung, wenn den Rechnungen die Geometrie des Radikal-Kations zugrunde gelegt wird, wobei aber auch hier eine Annihilierung keine

Tab. 2. Theoretische Hyperfeinaufspaltungen in $\mathrm{m} T$, berechnet für verschiedene Molekülgeometrien des Methylendioxybenzols mit Hilfe der INDO-Methode.

\begin{tabular}{|c|c|c|c|c|c|}
\hline $\begin{array}{l}\text { Me- } \\
\text { thode }\end{array}$ & 1 & 2 & 3 & 4 & exp. \\
\hline \multicolumn{6}{|c|}{ Verbindung 1 (Standardgeometrie) } \\
\hline $\begin{array}{l}a_{\mathrm{CH}_{2}} \\
a_{\mathrm{H}_{\alpha}} \\
a_{\mathrm{H}_{\beta}}\end{array}$ & $\begin{array}{r}2,156 \\
0,062 \\
-0,236\end{array}$ & $\begin{array}{r}2,112 \\
0,029 \\
-0,102\end{array}$ & $\begin{array}{r}2,026 \\
0,117 \\
-0,355\end{array}$ & $\begin{array}{r}2,276 \\
0,061 \\
-0,176\end{array}$ & $\begin{array}{ll}2,142 \\
0,0 \\
(-) \quad 0,481\end{array}$ \\
\hline \multicolumn{6}{|c|}{ Verbindung 1 a (Optimierte Geomet ie des Neutral-Moleküls) } \\
\hline $\begin{array}{l}a_{\mathrm{CH}_{2}} \\
a_{\mathrm{H}_{\alpha}} \\
a_{\mathrm{H}_{\beta}}\end{array}$ & $\begin{array}{r}3,313 \\
0,067 \\
-0,253\end{array}$ & $\begin{array}{r}3,157 \\
0,029 \\
-0,109\end{array}$ & $\begin{array}{r}3,094 \\
0,127 \\
-0,385\end{array}$ & $\begin{array}{r}3,338 \\
0,061 \\
-0,192\end{array}$ & $\begin{aligned} & 2,142 \\
& 0,0 \\
(-) & 0,481\end{aligned}$ \\
\hline \multicolumn{6}{|c|}{ Verbindung $1 \mathbf{b}^{-}$(Optimierte Geometrie des Ra dikal-Kations) } \\
\hline $\begin{array}{l}a_{\mathrm{CH}_{2}} \\
a_{\mathrm{H}_{\alpha}} \\
a_{\mathrm{H}_{\beta}}\end{array}$ & $\begin{array}{r}2,055 \\
0,098 \\
-0,303\end{array}$ & $\begin{array}{r}1,960 \\
0,040 \\
-0,131\end{array}$ & $\begin{array}{r}1,937 \\
0,175 \\
-0,461\end{array}$ & $\begin{array}{r}2,115 \\
0,088 \\
-0,234\end{array}$ & $\begin{array}{ll} & 2,142 \\
& 0,0 \\
(-) & 0,481\end{array}$ \\
\hline
\end{tabular}

a Die Zuordnung der Methoden ist in Tab. 1 beschrieben.
Verbesserung bringt. Die optimierte Geometrie des neutralen Moleküls verschlechtert die Ergebnisse.

\subsection{HMO-Methode}

Mit den von Streitwieser [14] angegebenen Hückel-Parametern für das Dioxol-Fragment

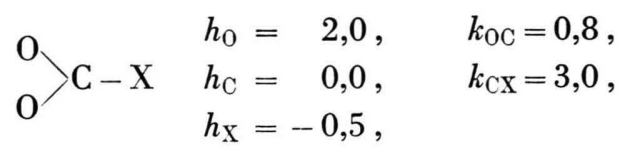

erhielten wir die in Tab. 3 angegebenen Energien der $\pi$-HOMO's und die Spin-Dichte $\varrho$ der RadikalKationen. Die Spin-Dichten wurden für die Verbindungen 1-3, bei denen das Energieniveau des HOMO's im Gegensatz zu 4 nicht entartet ist, zusätzlich nach der Methode von McLachlan [15] berechnet (s. Tab. 3)

$\varrho_{i}^{\prime}=\varrho_{i}+\lambda \beta \sum_{j}\left(\pi_{i j} c_{\text {HOMO }, j}^{2}\right) \quad(\lambda=1,2)$.

$\pi_{i j}$ ist die Atom-Atom-Polarisierbarkeit zwischen $\operatorname{dem} i$-ten und $j$-ten Atom (Einheit $\beta^{-1}$ ).

In Tab. 3 sind weiterhin die aus diesen SpinDichten über die McConnell-Beziehung [16]

$$
a_{i}=Q \varrho_{i} \quad(Q=-2,3 \mathrm{mT})
$$

berechneten Aufspaltungskonstanten der aromatischen Ring-Protonen angegeben.

Die Konstante $k$ in der Beziehung

$$
a_{\mathrm{CH}_{2}}=0,5 k \varrho \mathrm{x}
$$

für die Aufspaltungskonstante der Methylenprotonen wurde aus dem experimentellen $\alpha_{\mathrm{CH}_{2}}$ von 1 $(2,142 \mathrm{mT})$ und der Hückel-Spin-Dichte am Pseudoatom $\mathrm{X}$ zu 115,85 $\mathrm{mT}$ bzw. aus der entsprechenden McLachlan-Spin-Dichte zu 164,26 mT bestimmt. Die mit diesen Konstanten für 2, 3 und $\mathbf{4}$ erhaltenen Werte sind ebenfalls in Tab. 3 angegeben.

Wie man Tab. 3 entnimmt, werden sowohl die einfachen Hückel- als auch die nach McLachlan berechneten Spin-Dichten der Verbindungen 1-4 am Pseudoatom $\mathrm{X}$ in Übereinstimmung mit dem Experiment immer kleiner.

Die qualitative Diskussion der Spin-Dichten ist sehr einfach (s. Abbildung 5). Wegen der Nähe der Energieniveaus der $\pi$-HOMO's des Benzols $\left(\psi_{+1}\right.$ und $\left.\psi_{-1} ; E=\alpha+\beta\right)$ und der Dioxol-Gruppierung $\left(\varphi_{\text {Hомо }} ; E=\alpha+1,55 \beta\right)$ bestimmen diese MO's im wesentlichen die Gestalt der HOMO's von 1-4 und damit dann auch die Spin-Dichten der RadikalKationen. 

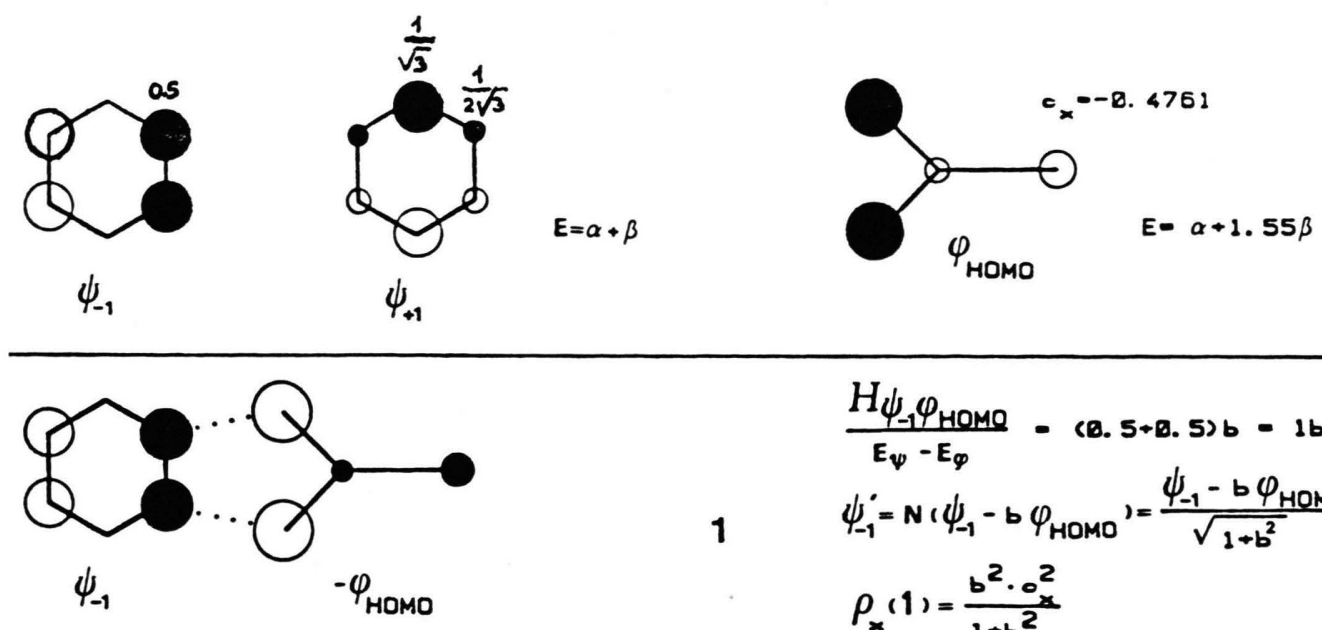

$$
\begin{aligned}
& \frac{H_{\psi_{-1}} \varphi_{\text {HOMO }}}{E_{\psi}-E_{\varphi}}-(0.5+B .5) b-1 b \\
& \psi_{-1}^{0}=N\left(\psi_{-1}-b \varphi_{\text {HOMO }}\right)=\frac{\psi_{-1}-b \varphi_{\text {HOMO }}}{\sqrt{1+b^{2}}} \\
& \rho_{x}(1)=\frac{b^{2} \cdot 0_{x}^{2}}{1+b^{2}}
\end{aligned}
$$

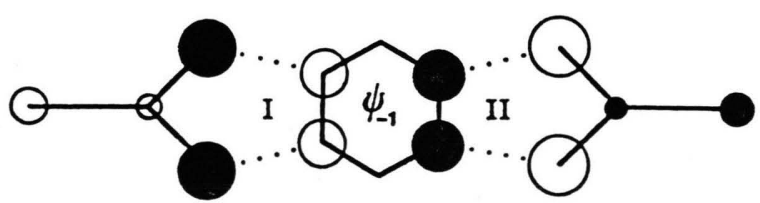

$\frac{H_{\psi_{-1}} \varphi_{\text {HOMO }}}{E_{\psi}-E_{\varphi}}=\frac{(0.5+D .5+0.5+0.5) b}{\sqrt{2}}=\sqrt{2 b}$
$\psi_{-1}^{\prime}=N\left(\psi_{-1}+\sqrt{2 b} \varphi_{\text {HOMO_- }}\right)=\frac{\psi_{-1}+\sqrt{2 b} \varphi_{\text {HOMO }}}{\sqrt{1+2 b^{2}}}$

$\varphi_{\text {HOMO- }}=\frac{1}{\sqrt{2}}\left\{\varphi_{\text {HOMO }}^{\text {I }}-\varphi_{\text {HOMO }}^{\text {II }}\right.$

$\rho_{x}(2)=\frac{(\sqrt{2} b)^{2}\left(c_{x} / \sqrt{2}\right)^{2}}{1+2 b^{2}}=\frac{b^{2} \cdot c_{x}^{2}}{1+2 b^{2}}$

$\rho_{x}(2)<\rho_{x}(1)$

\section{Abb. 5a.}

\begin{tabular}{|c|c|c|c|c|c|c|c|}
\hline \multirow{2}{*}{$\begin{array}{l}\text { Verbindung } \\
\text { und Energie } \\
\text { des HOMO's }\end{array}$} & \multicolumn{3}{|c|}{ Spin-Dichten } & \multicolumn{4}{|c|}{ Aufspaltungskonstanten } \\
\hline & & (1) & $(2)$ & & $(1)$ & $(2)$ & exp. ${ }^{c}$ \\
\hline 1 & $\varrho_{\alpha}$ & 0,014 & $-0,041$ & $a_{\mathrm{H}_{\alpha}}$ & $-0,032^{\mathrm{a}}$ & $0,094^{\mathrm{a}}$ & 0,040 \\
\hline & $\varrho_{\beta}$ & 0,144 & 0,187 & $a_{\mathrm{H}_{\beta}}$ & $-0,332^{\mathrm{a}}$ & $-0,429^{\mathrm{a}}$ & (-) 0,481 \\
\hline$E=\alpha+0,688 \beta$ & $0,5 \varrho \mathrm{x}$ & 0,018 & 0,013 & $a_{\mathrm{CH}_{2}}$ & $2,142^{\mathrm{b}}$ & $2,142^{\mathrm{b}}$ & 2,142 \\
\hline$E=\alpha \stackrel{2}{+} 0,518 \beta$ & $0,5 \stackrel{\varrho}{\varrho \mathrm{x}}$ & $\begin{array}{l}0,0 \\
0,010\end{array}$ & $\begin{array}{r}-0,062 \\
0,008\end{array}$ & $\begin{array}{l}a_{\mathrm{H}} \\
a_{\mathrm{CH}_{2}}\end{array}$ & $\begin{array}{l}0,0^{\mathrm{a}} \\
1,181^{\mathrm{b}}\end{array}$ & $\begin{array}{l}0,143^{\mathrm{a}} \\
1,309^{\mathrm{b}}\end{array}$ & $\begin{array}{l}0,102 \\
1,157\end{array}$ \\
\hline 3 & 0.5 & 0,090 & 0,087 & $a_{\mathrm{H}}$ & $-0,207 a$ & $-0,200^{\mathrm{a}}$ & $(-) 0,298$ \\
\hline$E=\alpha+0,594 \beta$ & $0,5 \varrho \mathrm{x}$ & 0,008 & 0,006 & $a_{\mathrm{CH}_{2}}$ & $0,979^{b}$ & $1,013^{b}$ & 0,891 \\
\hline$E=\alpha \stackrel{4}{+0,527 \beta}$ & $0,5 \varrho \mathrm{x}$ & 0,005 & - & $a_{\mathrm{CH}_{2}}$ & $0,607^{\mathrm{b}}$ & - & $0,54_{4}$ \\
\hline
\end{tabular}

Abb. 5. Wechselwirkungen zwischen den $\pi$-HOMO's des Benzols und des Dioxol-Fragmentes zur qualitativen Erklärung der relativen Größen der Aufspaltungskonstanten der Protonen von 1-4.

Tab. 3. HMOSpin-Dichten $\varrho$ und Aufspaltungskonstanten $a$ in $\mathrm{m} T$.

\footnotetext{
(1) Einfache Hückel-Rechnung.

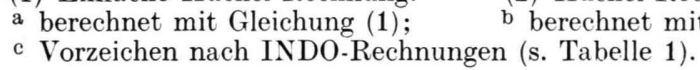

(2) Hückel-Rechnung mit Korrektur nach McLachlan.
} 


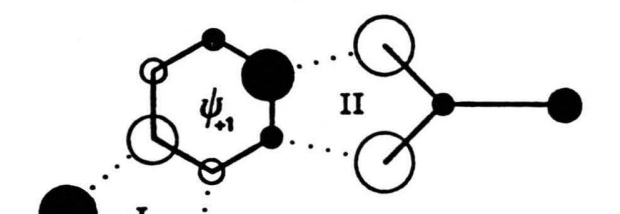

$$
\begin{aligned}
& \frac{H_{\psi_{+1}} \varphi_{\text {HOMO }}}{E_{\varphi}-E_{\varphi}}=\frac{1}{\sqrt{3}} \frac{(1+0.5+1+0.5) b}{\sqrt{2}}=\sqrt{\frac{3}{2} b} \\
& \psi_{+1}^{0}=N\left(\psi_{+1}+\sqrt{\frac{3}{2}} b \varphi_{\text {HOMO- }}\right)=\frac{\psi_{+1}+\sqrt{\frac{3}{2}} b \varphi_{\text {HOMO }}}{\sqrt{1+\frac{3}{2} b^{2}}} \\
& \rho_{x}(3)=\frac{(\sqrt{3 / 2} b)^{2}\left(C_{x} / \sqrt{2}\right)^{2}}{1+\frac{3}{2} b^{2}}=\frac{3}{4} \frac{b^{2} \cdot 0_{x}^{2}}{1+\frac{3}{2} b^{2}}
\end{aligned}
$$

3

$\varphi_{\text {HOMO- }}=\frac{1}{\sqrt{2}} \varphi_{\text {HOMO }}^{\mathrm{I}}-\varphi_{\text {HOMO }}^{\mathrm{II}}$

$$
\rho_{x}(3)<\rho_{x}(2)
$$

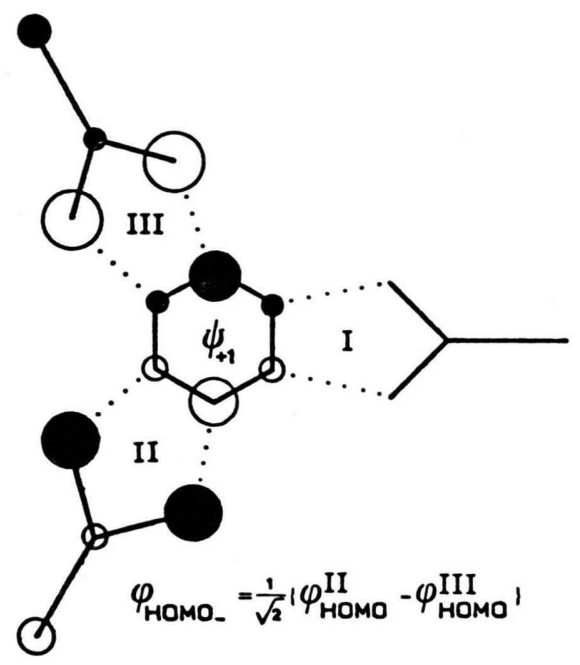

4

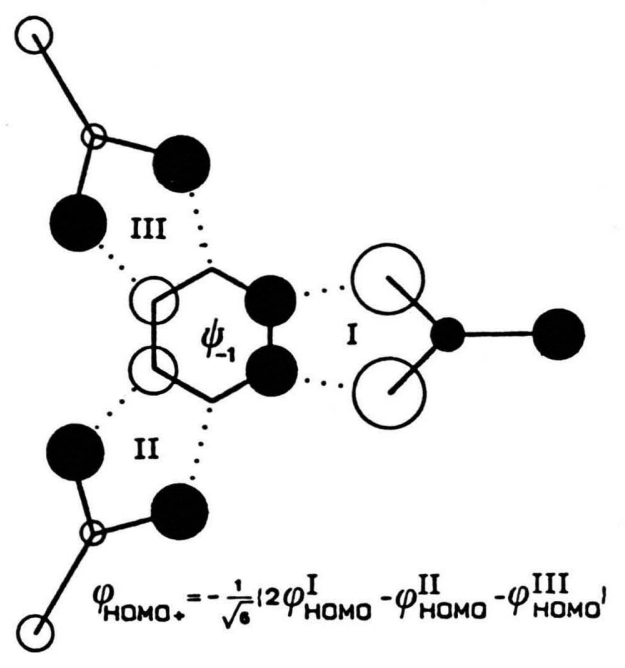

$\frac{H_{\psi_{11}} \varphi_{\text {HOMO}}}{E_{\varphi}-E_{\varphi}}=\frac{1}{\sqrt{3}}(1+0.5+1+0.5) b=\sqrt{\frac{3}{2} \mathrm{~b}}$

$\frac{H \psi_{-1} \varphi_{\text {HOMO }}}{E_{\psi}-E_{\varphi}}=\frac{1}{\sqrt{6}}(0.5 \cdot 2+0.5 \cdot 1+0.5 \cdot 1) b-\sqrt{\frac{3}{2}} b$

$\psi_{+1}^{:}=\frac{\psi_{+1}+\sqrt{\frac{\pi}{2}} b \varphi_{\text {HOMO }}}{\sqrt{1+\frac{3}{2} b^{2}}}$

$\rho_{x}(4)=\frac{1}{3} \frac{2\left(\sqrt{3 / 2} b \cdot a_{x}-\sqrt{2}\right)^{2}}{1+\frac{3}{2} b^{2}}=\frac{1}{2} \frac{b^{2} \cdot o_{x}^{2}}{1+\frac{3}{2} b^{2}}$

$\psi_{-1}^{\prime}=\frac{\psi_{-1}+\sqrt{\frac{3}{2} b} \varphi_{\text {номо }}}{\sqrt{1+\frac{3}{2} b^{2}}}$

$\rho_{x}(4)=\frac{1}{3} \frac{(4+1+1)\left(\sqrt{3 / 2} b \cdot 0_{x} / \sqrt{6}\right)^{2}}{1+\frac{3}{2} b^{2}}=\frac{1}{2} \frac{b^{2} \cdot a_{x}^{2}}{1+\frac{3}{2} b^{2}}$

Abb. 5b.

Verbindung 1: Hier kann $\varphi_{\text {Номо }}$ aus Symmetriegründen nur mit $\psi_{-1}$ wechselwirken. Das erklärt ohne weiteres die geringe Spin-Dichte am $\mathrm{C}_{a}$ im Vergleich zu $\mathrm{C}_{\beta}$, da $\psi_{-1}$ am $\mathrm{C}_{\alpha}$ einen Knoten besitzt.

Verbindung 2: Analog wie bei 1 kann die geringe Spin-Dichte am nichtsubstituierten aromatischen CAtom erklärt werden. Die im Vergleich zu 1 gerin- gere Spin-Dichte ist eine Folge der größeren Delokalisierung des ungepaarten Elektrons über das gesamte $\pi$-System (der Normierungsfaktor ist kleiner als bei 1).

Verbindung 3: Die Spin-Dichten an den nichtsubstituierten Ring-C-Atomen sind jeweils etwas kleiner als im Fall von 1, da hier das HOMO im 
wesentlichen durch $\psi_{+1}$ repräsentiert wird. Da das symmetrieadaptierte $\varphi_{\text {HOMO }}$ der beiden DioxolFragmente etwas geringer mit $\psi_{+1}$ wechselwirkt als es für 2 der Fall ist, erklärt sich auch, daß $\varrho_{\mathrm{X}}(3)<\varrho_{\mathrm{X}}(2)$ ist.

Verbindung 4: Hier wird die Entartung von $\psi_{+1}$ und $\psi_{-1}$ nicht aufgehoben. Sowohl $\psi_{+1}$ als auch $\psi_{-1}$ mischen jeweils gleichermaßen mit dem symmetrieadaptierten $\mathrm{HOMO} \varphi_{\mathrm{HOMO}}+$ bzw. $\varphi_{\mathrm{HOMO}}-\cdot$ Die „Ankopplung" von $\varphi_{\mathrm{HOMO}}$ - an $\psi_{+1}$ ist im Fall von 4 und 3 identisch. Die geringere Spin-Dichte am Pseudoatom $X$ in 4 verglichen mit 3 ist die Folge davon, daß man in 4 über drei Zentren mitteln muß.

\section{Experimenteller Teil}

\subsection{Herstellung der Reaktionskomponenten}

Zur Erzeugung der Radikal-Kationen wurde versucht, die Methylendioxybenzol-Derivate in Schwefelsäure (1N), wie in [6] beschrieben, zu lösen. Da sie in Schwefelsäure aber nicht vollständig löslich sind, wurden die Derivate zunächst in Aceton gelöst und dann die Schwefelsäure unter kräftigem Rühren hineingeleitet. Das Verhältnis Aceton zu Schwefelsäure wurde dabei entsprechend der Löslichkeit der zu untersuchenden Verbindungen variiert. Cer(IV) sulfat (Merck, Darmstadt) wurde ebenfalls in Schwefelsäure (1N) unter Rühren gelöst. Die Konzentrationen der Reaktionskomponenten sowie die Mischungsverhältnisse (V/V) Aceton : Schwefelsäure sind in Tab. 4 wiedergegeben.

\subsection{Erzeugung der Radikal-Kationen}

Zur Erzeugung der Radikal-Kationen wurden die oben beschriebenen Reaktionskomponenten in einer Mischzelle aus Quarz mit angeschmolzener Flachküvette (Bruker Analytische Meßtechnik, Karlsruhe)

Tab. 4. Konzentrationen und Mischungsverhältnis (V/V) der Reaktionskomponenten.

\begin{tabular}{lccll}
\hline $\begin{array}{l}\text { Konzentration } C \\
\text { in mol/l }\end{array}$ & \multicolumn{3}{c}{ Verbindung } \\
\hline & $\mathbf{1}$ & $\mathbf{2}$ & $\mathbf{3}$ & $\mathbf{4}$ \\
$C_{\text {Derivat }}$ & 0,01 & 0,003 & 0,01 & 0,002 \\
$C_{\text {Cer(IV)sulfat }}$ & 0,01 & 0,005 & 0,01 & 0,005 \\
Mischungsverhältnis (V/V) & & & \\
Aceton: Schwefelsäure & $\mathbf{1}: 9$ & $\mathbf{1}: 2$ & $2: 5$ & $1: 1$ \\
\hline
\end{tabular}

schnell gemischt. Dabei oxidierte Cer(IV) sulfat das entsprechende Methylendioxybenzol-Derivat in der Mischkammer zum Radikal-Kation, welches dann beim Durchfließen der Flachküvette ESR-spektroskopisch untersucht wurde.

\subsection{Meßbedingungen}

Die ESR-Spektren der Radikal-Kationen wurden in 1. Ableitung in einem X-Band-Spektrometer Typ ER 420 (Bruker Analytische Meßtechnik, Karlsruhe) vermessen. Das Magnetfeld wurde mit $100 \mathrm{kHz}$ moduliert und das Detektorsignal im $100 \mathrm{kHz}-$ Kanal verstärkt. Die Mikrowellenleistung wurde so gewählt, daß Sättigungserscheinungen nicht auftraten. Der Flachküvettenteil der Mischzelle befand sich in einem Hohlraumresonator vom Typ $\mathrm{H}_{102}$. Magnetfeldeichmarken wurden mit Hilfe eines Kernresonanzoszillators und einer Kernresonanzsonde, die sich in unmittelbarer Nähe des Resonators befand, erzeugt. Gleichzeitig wurde die Mikro. wellenfrequenz mit Hilfe eines Frequenzzählers gemessen. Aufgrund der angewandten Durchflußtechnik [17] waren die Konzentrationen der erzeugten Radikale in der Meßküvette erheblich niedriger als die Anfangskonzentrationen der einzelnen Reaktionskomponenten. Daher mußten bei den Derivaten 1 und 4 höhere Mikrowellenleistungen und Modulationsamplituden benutzt werden, um ein günstiges Signal-Rausch-Verhältnis zu erreichen. Die für die Messungen bei Raumtemperatur verwendeten Parameter sind in Tab. 5 zusammengestellt.

\section{4. g-Faktoren der Methylendioxybenzol-Derivate}

Die $g$-Faktoren der vermessenen Derivate sind in den meisten Fällen gegenüber dem $g$-Faktor des

Tab. 5. Bedingungen für die ESR-Spektroskopie der Radikal-Kationen der Methylendioxybenzol-Derivate bei Raumtemperatur.

\begin{tabular}{lllll}
\hline Parameter & \multicolumn{4}{l}{ Verbindung } \\
\hline & $\mathbf{1}$ & $\mathbf{2}$ & $\mathbf{3}$ & $\mathbf{4}$ \\
$\begin{array}{l}\text { Modulations- } \\
\text { amplitude in mT }\end{array}$ & 0,05 & 0,005 & 0,0032 & 0,05 \\
$\begin{array}{l}\text { Mikrowellenleistung } \\
\text { in mW }\end{array}$ & 50 & 0,4 & 5 & 1,5 \\
$\begin{array}{l}\text { Verstärkung } \\
\text { Zeitkonstante in s }\end{array}$ & $1 \cdot 10^{6}$ & $4 \cdot 10^{4}$ & $4 \cdot 10^{4}$ & $3,2 \cdot 10^{6}$ \\
Sweepzeit in s & 2000 & 500 & 500 & 1000 \\
\hline
\end{tabular}


Tab. 6. ESR-Parameter der Methylendioxybenzol-Derivate.

\begin{tabular}{lllll}
\hline Derivat & $g$-Faktor & \multicolumn{4}{c}{ Hyperfeinaufspaltung in $\mathrm{mT}^{\mathrm{a}}$} \\
\hline & & $a_{\mathrm{CH}_{2}}$ & $a_{\mathrm{H}}$ & \\
$\mathbf{1}$ & 2,00395 & 2,142 & $0,0\left(a_{\mathrm{H}_{\alpha}}\right) ;$ & $0,481\left(a_{\mathrm{H}_{\beta}}\right)$ \\
$\mathbf{2}$ & 2,00440 & 1,157 & 0,102 & \\
$\mathbf{3}$ & 2,00394 & 0,891 & 0,298 & \\
$\mathbf{4}$ & $\mathbf{2 , 0 0 3 9 3}$ & 0,544 & & \\
\hline
\end{tabular}

a Experimentelle Werte für Verbindung 1 nach Lit. [6]: $a_{\mathrm{CH}_{2}}=2,19 \mathrm{mT} ; a_{\mathrm{H}_{\alpha}}=0,04 \mathrm{mT} ; a_{\mathrm{H}_{\beta}}=0,49 \mathrm{mT}$.

[1] F. Dallacker, W. Edelmann u. A. Weiner, Liebigs Ann. Chem. 719, 112 (1968).

[2] F. Dallacker u. A. Weiner, Liebigs Ann. Chem. 725, 99 (1969).

[3] F. Dallacker u. J. Krause, Liebigs Ann. Chem. 1975, 611.

[4] G. Klopman, J. Amer. Chem. Soc. 90, 223 (1968).

[5] L. Salem, J. Amer. Chem. Soc. 90, 543 (1968); J. Amer. Chem. Soc. 90, 553 (1968). - K. Fukui, Top. Curr. Chem. 15, 1 (1970); Acc. Chem. Res. 4, 57 (1971). - I. Fleming, Frontier Orbitals and Organic Chemical Reactions, John Wiley, London 1976. - J. Fleischhauer, A. N. Asaad, W. Schleker u. H.-D. Scharf, Liebigs Ann. Chem. 1981, 306.

[6] W. T. Dixon u. D. Murphy, J. Chem. Soc., Perkin II $1976,1823$.

[7] S. Ma, G. Raabe u. J. Fleischhauer, Z. Naturforsch. 36 a, 521 (1981).

[8] J. A. Pople, D. L. Beverigde u. P. A. Dobosh, J. Chem. Phys. 47, 2026 (1967); J. Amer. Chem. Soc. 90, 4201 (1968). freien Elektrons geringfügig erhöht (s. Tabelle 6). Eine Ausnahme bildet Verbindung 2. Für Methylendioxybenzol wurde derselbe $g$-Faktor ermittelt, der schon in [6] mitgeteilt ist.

Wir danken dem Fonds der Chemischen Industrie (J.F.) und der Deutschen Forschungsgemeinschaft (K.G.) für die gewährte Unterstützung und dem Rechenzentrum der RWTH Aachen für die Bereitstellung von Rechenzeit.

[9] J. J. Kaufman, Int. J. Quant. Chem. 6, 231 (1972).

[10] J. Tino u. V. Klimo, Chem. Phys. Lett. 25, 427 (1974).

[11] A. T. Amos u. L. C. Snyder, J. Chem. Phys. 41, 1773 (1964). - H. Thiele, Dissertation, RWTH Aachen 1978.

[12] D. L. Beveridge u. P. A. Dobosh, J. Chem. Phys. 48, 5532 (1968).

[13] R. C. Bingham, M. J. S. Dewar u. D. H. Lo, J. Amer. Chem. Soc. 97, 1285 (1975).

[14] A. Streitwieser, Molecular Orbital Theory for Organic Chemists, John Wiley, New York 1961.

[15] A. D. McLachlan, Mol. Phys. 3, 233 (1960).

[16] H. M. McConnell, J. Chem. Phys. 24, 632 (1956); J. Chem. Phys. 24, 764 (1956). - H. M. McConnell u. D. B. Chestnut, J. Chem. Phys. 27, 984 (1957); J. Chem. Phys. 28, 107 (1958).

[17] W. T. Dixon u. R. O. C. Norman, J. Chem. Soc. 1964, 4850 . 